\title{
240 Blood oxygen-sensitive ssfp imaging for probing the myocardial perfusion reserves of patients with coronary artery disease: a feasibility study
}

\author{
Rohan Dharmakumar*1, Jordin Green ${ }^{2}$, Jacqueline Flewitt ${ }^{3}$, \\ Matthias Voehringer ${ }^{3}$, Neil G Filipchuk ${ }^{3}$, Debiao $\mathrm{Li}^{1}$ and \\ Matthias G Friedrich ${ }^{3}$
}

\author{
Address: ${ }^{1}$ Northwestern University, Chicago, IL, USA, ${ }^{2}$ Siemens Medical Solutions, Calgary, AB, Canada and ${ }^{3}$ University of Calgary, Calgary, AB, \\ Canada \\ * Corresponding author
}

from I th Annual SCMR Scientific Sessions

Los Angeles, CA, USA. I-3 February 2008

Published: 22 October 2008

Journal of Cardiovascular Magnetic Resonance 2008, I0(SuppI I):AI0I doi:I0.II86/I532-429X-I0-SI-AI0I

This abstract is available from: http://jcmr-online.com/content/I0/SI/AI0I

(c) 2008 Dharmakumar et al; licensee BioMed Central Ltd.

\section{Introduction}

Myocardial oxygen deficits secondary to coronary artery stenosis may be detected with blood-oxygen-leveldependent (BOLD) MRI. Similar to the first-pass perfusion technique, BOLD MRI identifies functionally significant coronary artery stenosis based on regional myocardial signal intensity differences at stress (pharmocological vasodilation). While the advantages of BOLD MRI have been long recognized, long scan times, poor image quality and/or oxygen sensitivity have limited the clinical utility of this technique. BOLD MRI based on steady-state free precession (SSFP) is a promising approach to overcoming many of these obstacles. It has been used to detect regional myocardial oxygen differences in canine models with experimental coronary artery stenosis.

\section{Purpose}

This work investigates whether SSFP BOLD MRI can detect regional myocardial perfusion deficits in patients who have been tested positive for coronary artery disease via $\mathrm{x}$ ray angiography and Thallium SPECT.

\section{Methods \\ Data acquisition}

Nine patients with coronary artery disease underwent CMR studies using procedures and protocols approved by our institution. Studies were performed using a Siemens Avanto $1.5 \mathrm{~T}$ scanner. Following whole-heart manual shim, a mid-ventricular slice was identified. This was followed by acquisitions of 2D SSFP images at mid-ventricle during rest and stress. First-pass perfusion scans at rest and stress, and late-enhancement scans covering the mid-ventricular slice of interest were also obtained. Adenosine studies were performed 3 minutes after starting an intravenous infusion of Adenosine $(140 \mu \mathrm{g} / \mathrm{kg}$ body weight/ min). Imaging parameters for 2D SSFP scans were: $T_{R} / T_{E}=$ $5.8 / 2.9 \mathrm{~ms}$; flip angle $=90^{\circ}$; single-breath hold $(13-20 \mathrm{~s})$ with retrospective gating (20 cardiac phases); and voxel size $=1.5 \times 1.5 \times 5 \mathrm{~mm}^{3}$.

\section{Data analysis}

End-systolic frames obtained before and during Adenosine were analyzed in a segmental fashion using the AHA 6-segment model. Based on Thallium SPECT, segments were classified as healthy, mildly affected, severely affected, or infarcted. Infarcted segments (fixed defect on SPECT and positive on late enhancement images) were excluded from further analysis. Segmental SSFP signal 
intensities at rest and stress were measured and the signal intensity ratio (Stress/Rest) was calculated. One-way ANOVA with Tukey post-hoc analysis was used to determine the statistical significance of regional differences.

\section{Results}

Fig. 1 shows representative example mid-ventricular short axis SSFP-based myocardial BOLD images obtained under rest (A) and stress (D) from a patient with 70\% narrowing of the left anterior descending artery. For reference, midventricular short axis first-pass perfusion (B, rest and $\mathrm{E}$, stress) and SPECT (C, rest and F, stress) images are also shown. Note the discriminating signal loss in the stress images (relative to rest) in the anterior zones of the myocardium and its close correspondence to first pass and SPECT images. Statistical results (Fig. 2) show that there are significant differences in Stress/Rest values computed from healthy, mildly and severely affected segments ( $\mathrm{p}<$ 0.05).

\section{Discussion}

In the absence of coronary artery stenosis, pharmacological stress is expected to increase basal myocardial perfusion and vascular oxygenation. The progression of coronary artery stenosis reduces this vasodilatory capacity, resulting in oxygenation changes in corresponding myocardial territories. Since SSFP signals are directly dependent on blood oxygenation, the signal intensity ratios between rest and stress conditions can be used to identify ischemic territories of the myocardium.

\section{Conclusion}

This work demonstrated that 2D SSFP CMR at $1.5 \mathrm{~T}$ may provide a robust non-contrast technique capable of identifying regional myocardial oxygen deficits in patients with known coronary artery stenosis. Further studies are warranted to establish the sensitivity and specificity of the technique.

\section{SSFP BOLD}

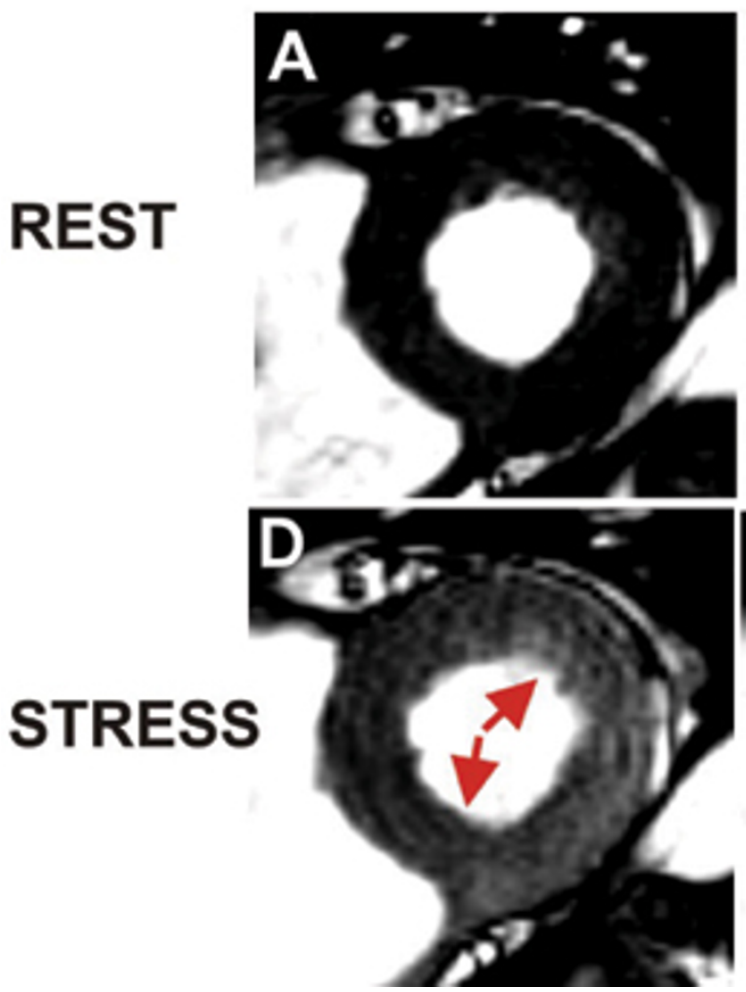

FPP
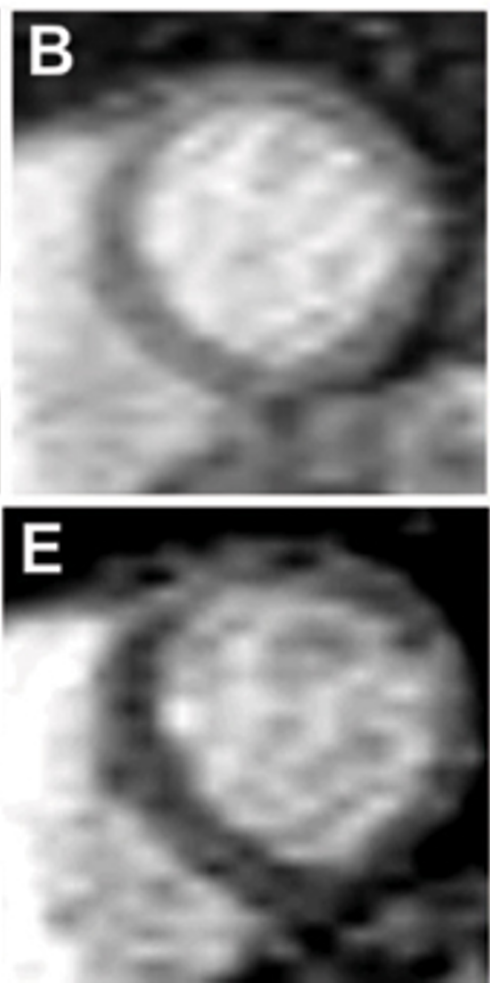

\section{Thallium SPECT}
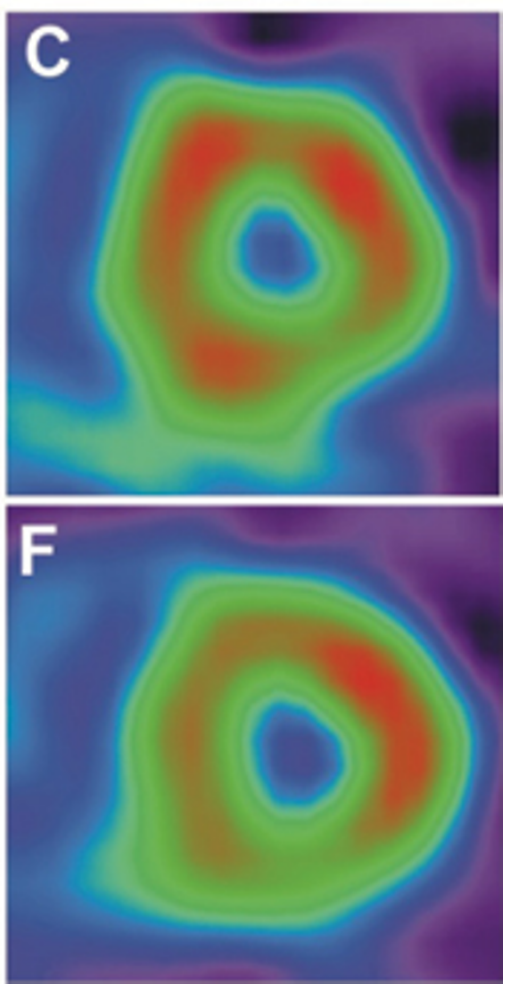

\section{Figure I}

Mid-ventricular short-axis SSFP BOLD, First-pass perfusion (FPP), and Thallium SPECT images obtained from a patient with $70 \%$ stenosis of the left anterior descending (LAD) coronary artery at rest and stress. For each imaging method, the windowing and leveling of images obtained at rest and stress are the same. The myocardial signal in the rst BOLD, FP, and SPECT images are relatively homogenous. However, under stress, the territory supplied by the LAD (larger arc subtended by arrow) does not increase in the BOLD images as expected. This pattern of regional signal differences is also evident in the FP and SPECT images. 


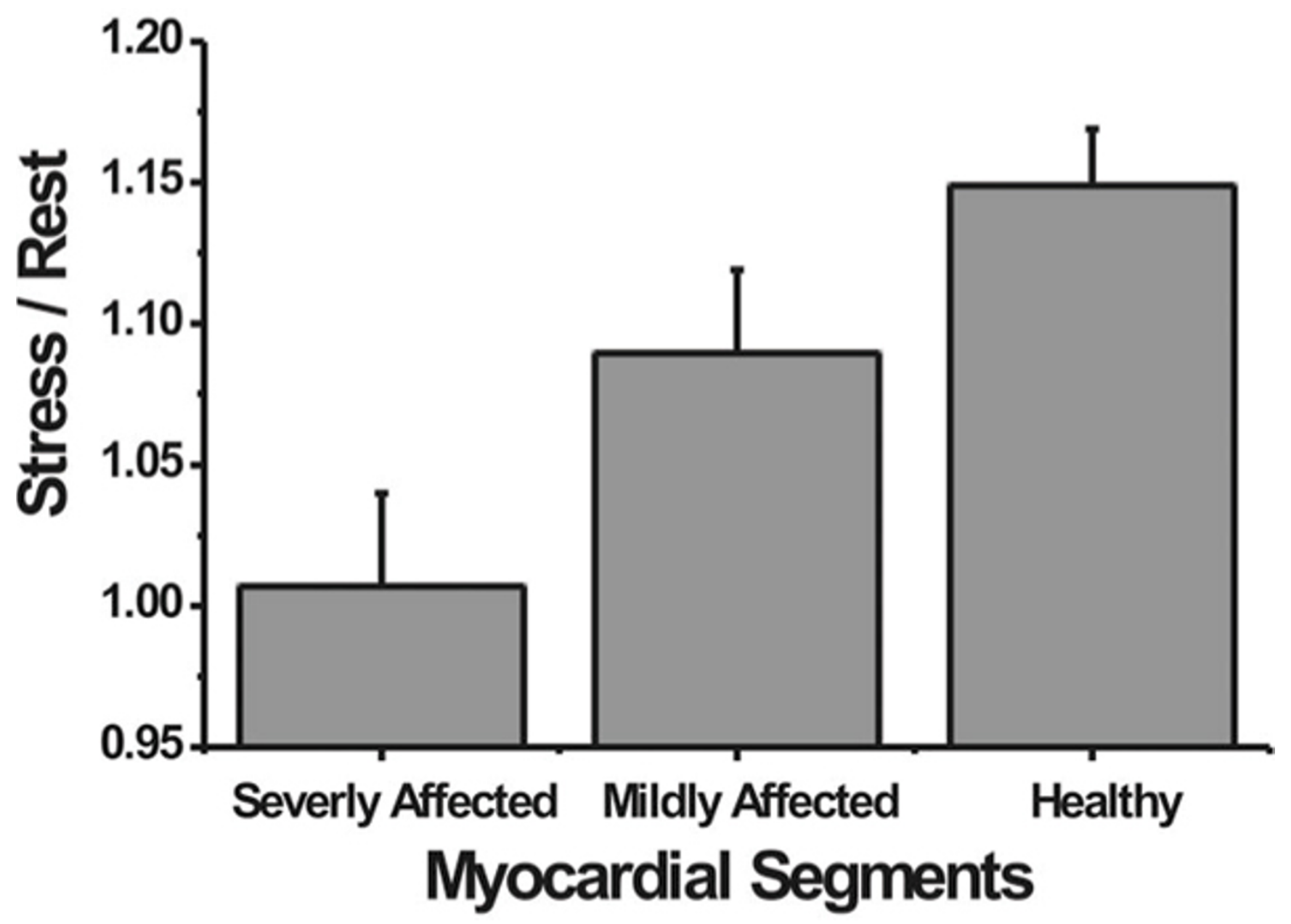

Figure 2

Statistical results from the myocardial BOLD signal analysis. Statistically significant differences in Stress/Rest values obtained from affected segments are lower, in comparison with healthy segments, consistent with previous findings in animals that SSFP signals obtained under pharmacological stress are significantly reduced in myocardial territories supplied by stenotic arteries.

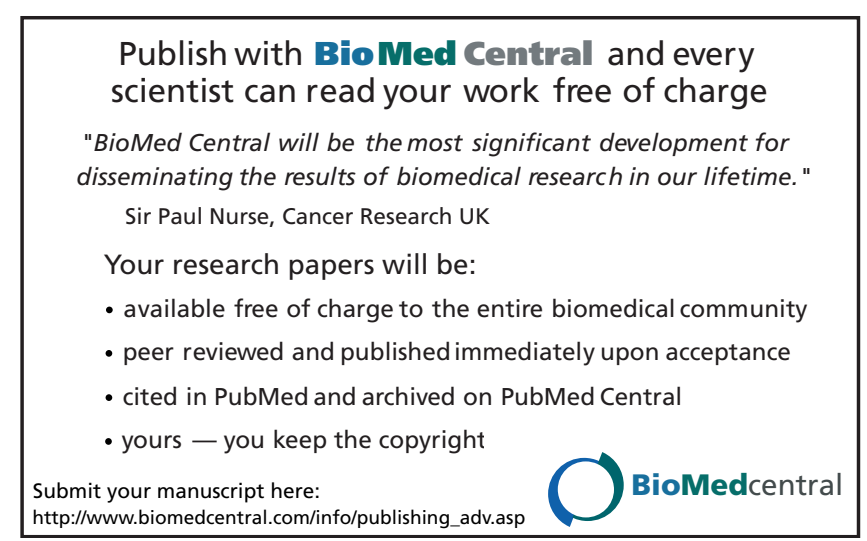

